\title{
Determinant of a Successful Case: Clinical Changes or Cephalometric Readings? Class II Division 1 Correction Using Forsus Fatigue Resistant Device
}

\section{Ambesh Kumar Rai \\ Department of Orthodontics, Al Reef Dental and orthodontic Centre, Ras Al khaimah, United Arab Emirates}

*Corresponding author: Ambesh Kumar Rai, BDS, MDS, FAGE Orthodontist, Department of Orthodontics, Al Reef Dental and orthodontic Centre, Ras Al khaimah, United Arab Emirates, Tel: +971 554841061; E-mail: drambeshrai@gmail.com

Rec Date: Oct 15, 2014; Acc Date: Nov 14, 2014; Pub Date: Nov 22, 2014

Copyright: (c) 2014 Rai AK. This is an open-access article distributed under the terms of the Creative Commons Attribution License, which permits unrestricted use, distribution, and reproduction in any medium, provided the original author and source are credited.

\begin{abstract}
Success is individualistic and orthodontics is one of the best places in dentistry to experience this. What determines the success of a treatment- a satisfied patient or the cephalometric readings is an intriguing question that would cross the mind of every reflecting orthodontist, time and again when they look at their treated cases.

Presented in this article is such a case of a class II division 1 patient treated with Forsus fatigue resistant device whose treatment whether judged a success or a failure would make us ponder what should we focus more on- the facial changes or cephalometic readings.
\end{abstract}

Keywords: Fixed functional appliance; Forsus; Growth modulation; Class II malocclusion

\section{Introduction}

When an orthodontic treatment planning for a functional appliance is done, rarely do we talk on cephalometric goals with our patient, which ironically becomes a topic of much discussion among orthodontist when discussing the success of a treatment in retrospect. This report presents such a case where marked facial changes seen fell short of cephalometric goals. This article aims to raise a question whether over emphasis on cephalometrics is actually justified when clinical results look acceptable while judging the treatment to be a success or failure.

The management of class II depends mainly on the severity of the problem and the age at which the patient presents. Numerous orthodontic techniques and appliances have been introduced for its treatment for this reason. Correction of skeletal class II during growth period often involves the use of functional appliances [1]. Functional appliances can simply be defined as appliances that bring about change in function of orofacial musculature to produce change in orofacial complex.

Functional appliances can broadly be classified in to two types the removable appliances (those which can be removed and replaced by the patient)-used when much of growth is remaining and fixed functional appliances (those which cannot be removed or replaced by the patient and needs a clinician to do so) - used towards the end of growth phase. Fixed functional appliances like Herbst, Jasper jumper, Forsus fatigue resistant device (FRD) require minimum patient compliance and can produce satisfactory functional and esthetic correction harnessing the residual growth remaining hence, their increasing popularity [2].

The Forsus FRDTM (3M Unitek Corp, Monrovia, California) offers the advantages of being robust, easy to use clinically, ease of installation and activation, produce predictable result in non compliant and handicapped patients, harnesses residual growth and shortens treatment timing. From the patient's perspective, it allows freedom of jaw movement, less tissue impingement and ease of maintaining oral hygiene. This article presents the case report of a patient treated with this appliance.

\section{Case report}

A 12-year-old male reported to the Department of Orthodontics, SDM Dental College and Hospital, Dharwad with the chief complaint of forwardly placed upper front teeth and the patient's parents were visibly distressed over the teasing vested to their child who was often teased as 'rabbit' or 'bugs bunny' by children. The medical history revealed the patient had repeated episodes of apthous ulcers in the past and has been on medications for the same off and on.

On extraoral examination, he had a symmetric, mesoprosopic face and a mesocephalic head form. Profile was convex, with incompetent lips, acute nasolabial angle and a deep mentolabial sulcus, proclined maxillary central inclisors and a concomitant lower lip trap. Figure 1 (a-d) shows his pretreatment extraoral photographs.

VTO (Visual treatment objective when elicited was positive). VTO is a clinical chair side technique to show changes anticipated in the course of growth and treatment. If the patient's profile improves by repositioning the mandible the patient is likely to benefit from functional appliance treatment and the VTO so elicited is said to be positive.

Intraoral examination revealed end on molar relationship bilaterally, canine relation cannot be assesses as canine were in transitional stage. He exhibited an increased overjet of $14 \mathrm{~mm}$ and a deep overbite of $10 \mathrm{~mm}$. Upper and lower midlines were coinciding with the facial midlines. Figures 2 (a-d) and 3 (a-c) shows his pretreatment intraoral and occlusal photographs respectively. Lateral cephalometric analysis showed skeletal class II malocclusion with retrognathic mandible (Table 1). He had an average growth pattern. 
Citation: Rai AK (2014) Determinant of a Successful Case: Clinical Changes or Cephalometric Readings? Class II Division 1 Correction Using Forsus Fatigue Resistant Device. Dentistry 4: 266. doi:10.4172/2161-1122.1000266

Page 2 of 7
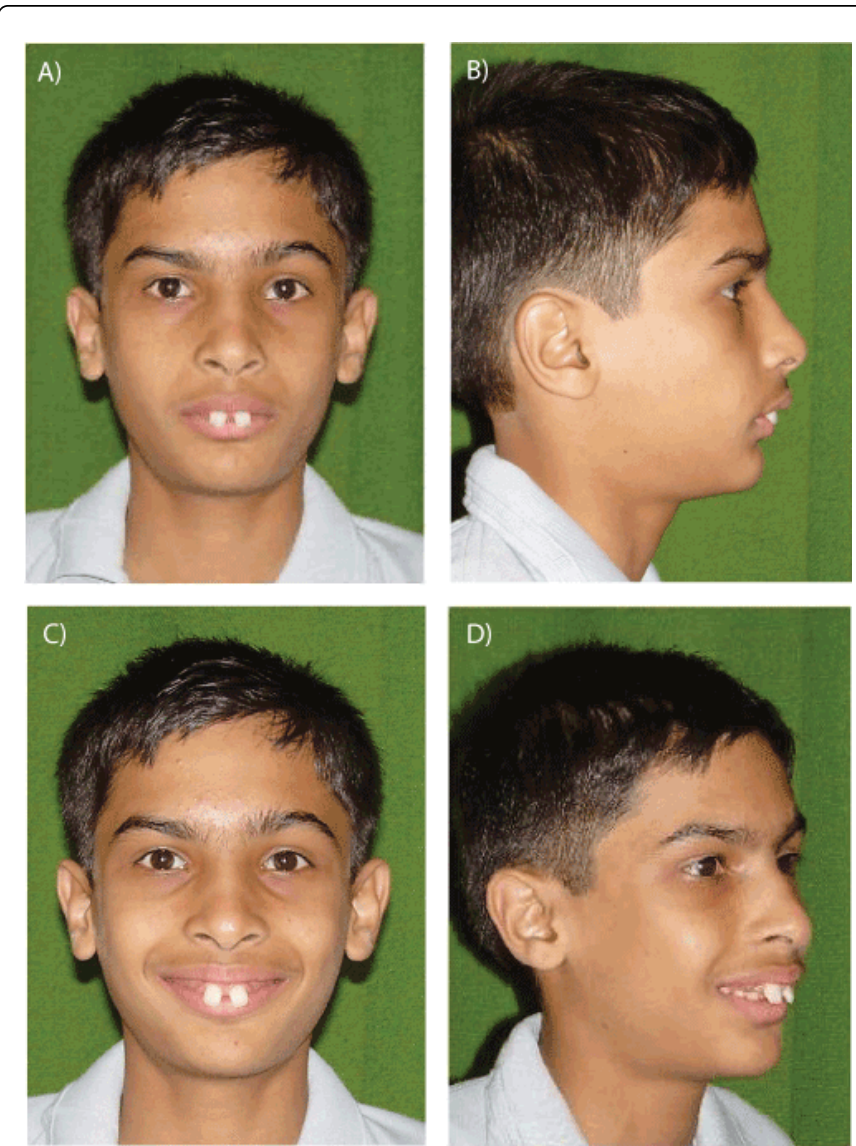

Figure 1: Pretreatment extraoral photographs

Maxillary incisors were proclined and forwardly placed while the mandibular incisors were upright. Cervical Vertebrae Maturity Index (CVMI) revealed that 60 to $65 \%$ of adolescence growth was expected (CVMI Stage II). CVMI is a technique that is used to assess skeletal age using lateral cephalometric radiographs.

\section{Treatment plan}

The prime concern of the orthodontist at this stage was to improve the appearance of the patient and potentially unlock the mandible by aligning the maxillary lateral incisors. Reassessment was to be done after that whether a functional, camoflague or surgical line of treatment would follow.

\section{Treatment progress}

Considering the palatally inclined 12 and 22 which may possibly be restricting mandibular growth it was decided to perform a prefunctional orthodontics to align the laterals to facilitate possible mandibular relocation and growth using Mulligan's $2 \times 4$ appliance set up.

0.022 inch slot metal brackets with MBT versatile+brackets $(3 \mathrm{M}$ Unitek Corporation, Monrovia, California, USA) were used for the strap up. An in initial $2 \times 2$ Mulligan's intrusion arch to align and intrude the maxillary central incisors. This was followed by $2 \times 4$ intrusion arch to align and intrude the laterals also (Figure 4).
A stepwise full mouth strap up followed to align upper and lower arches using $0.014,0.016,0.018$ inch HANT (heat activated nickle titanium) followed by $0.019 \times 0.025$ inch HANT and subsequently $0.019 \times 0.025$ inch Stainless steel wire to complete leveling and aligning of the arches which lasted for a total of 13 months.
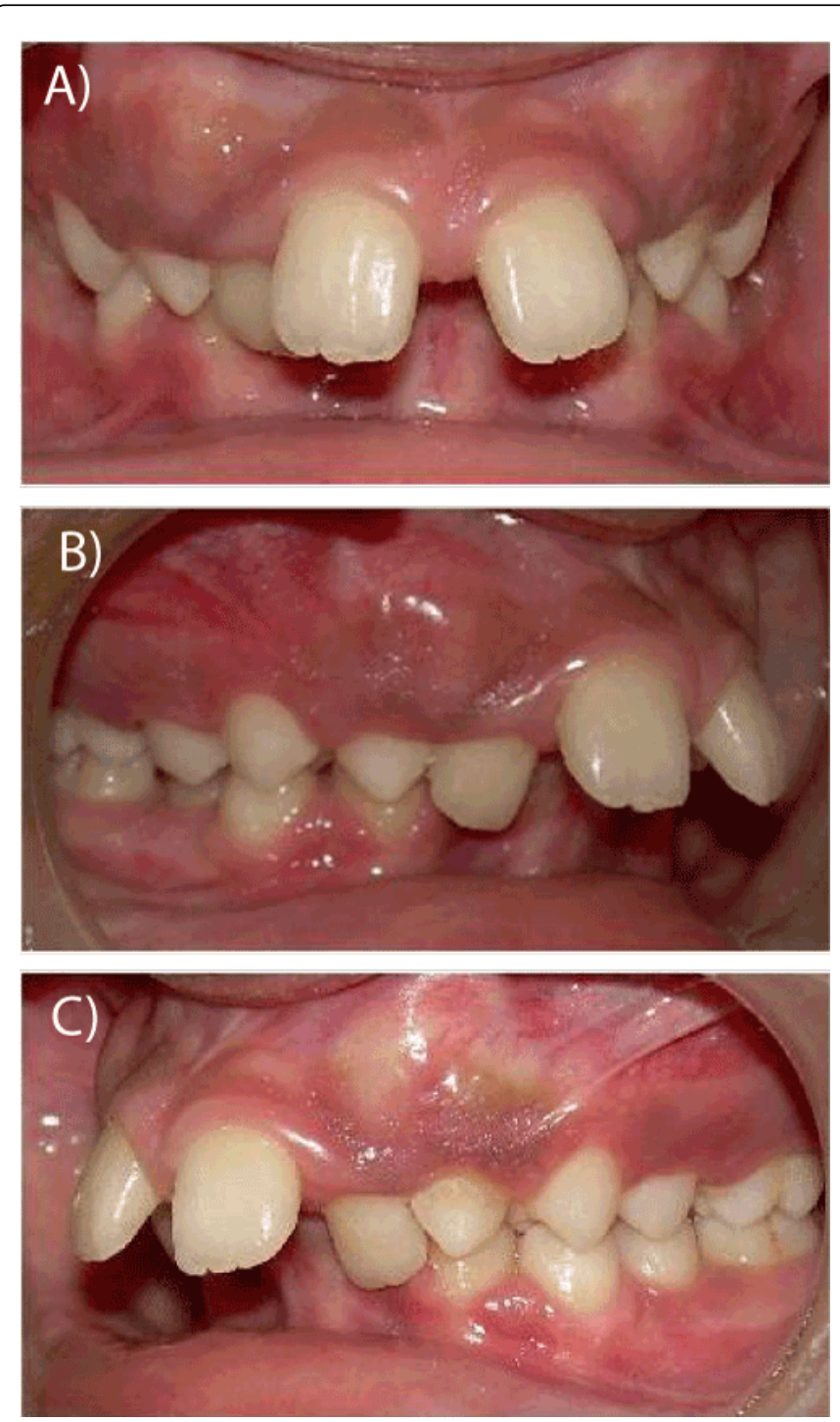

Figure 2: Pretreatment intraoral photographs

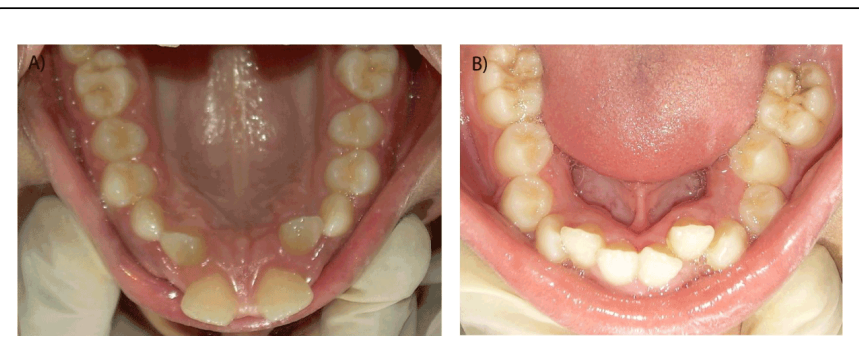

Figure 3: Pretreatment occlusal photographs 


\begin{tabular}{|l|l|l|l|}
\hline Cephalometric parameter & Pretreatment & $\begin{array}{l}\text { Postfunction } \\
\text { al }\end{array}$ & $\begin{array}{l}\text { Posttreatmen } \\
\mathbf{t}\end{array}$ \\
\hline SNA & $88^{\circ}$ & $86^{\circ}$ & $85^{\circ}$ \\
\hline SNB & $77^{\circ}$ & $79^{\circ}$ & $79^{\circ}$ \\
\hline ANB & $9^{\circ}$ & $7^{\circ}$ & $6^{\circ}$ \\
\hline U1-SN & $120^{\circ}$ & $112^{\circ}$ & $109^{\circ}$ \\
\hline L1-MP & $85^{\circ}$ & $109^{\circ}$ & $109^{\circ}$ \\
\hline U1-A Pog & $47^{\circ}, 12 \mathrm{~mm}$ & $38^{\circ}, 5 \mathrm{~mm}$ & $24^{\circ}, 1 \mathrm{~mm}$ \\
\hline L1-A Pog & $10^{\circ},-8 \mathrm{~mm}$ & $33^{\circ}, 1 \mathrm{~mm}$ & $40^{\circ}, 5 \mathrm{~mm}$ \\
\hline IIA & $130^{\circ}$ & $115^{\circ}$ & $118^{\circ}$ \\
\hline Bjork sum & $388^{\circ}$ & $434^{\circ}$ & $387^{\circ}$ \\
\hline Sn-GoGn & $25^{\circ}$ & $24^{\circ}$ & $24^{\circ}$ \\
\hline Y axis & $68^{\circ}$ & $66^{\circ}$ & $66^{\circ}$ \\
\hline Naso Labial angle & $105^{\circ}$ & $115^{\circ}$ & $113^{\circ}$ \\
\hline Harvold unit length difference & $15 \mathrm{~mm}$ & $27 \mathrm{~mm}$ & $27 \mathrm{~mm}$ \\
\hline
\end{tabular}

Table1: Cephalometric values of the patient

The case was re-evaluated to decide the further course of treatment at the end of leveling and aligning. The important factors mainly considered deciding the further course of treatment were:

i) No significant mandibular relocation took place despite removal of potential interference by maxillary lateral incisors, hence, an increased overjet and overbite remained to be addressed.

ii) The patient had a vertical and antero posterior maxillary excess which was seen as increased gumminess during smiling and a markedly obvious overjet.

iii) The patient had minimal growth remaining.

In light of aforementioned the parent's and the patient were given three options being:

a) Dental camouflage with upper first premolars extraction and implant assisted intrusion and retraction.

b) Surgical treatment with upper premolar extraction and premaxillary set back and impaction to correct the gumminess and overjet. Rhinoplasty might be needed to further enhance appearance.

c) Non extraction treatment using functional appliance to advance the mandible and restrict maxillary growth. It was further explained to the parents that the result achieved would be more of dentoalveolar correction and absolute resolution of skeletal problems might not be possible owing to the limited amount of growth remaining.
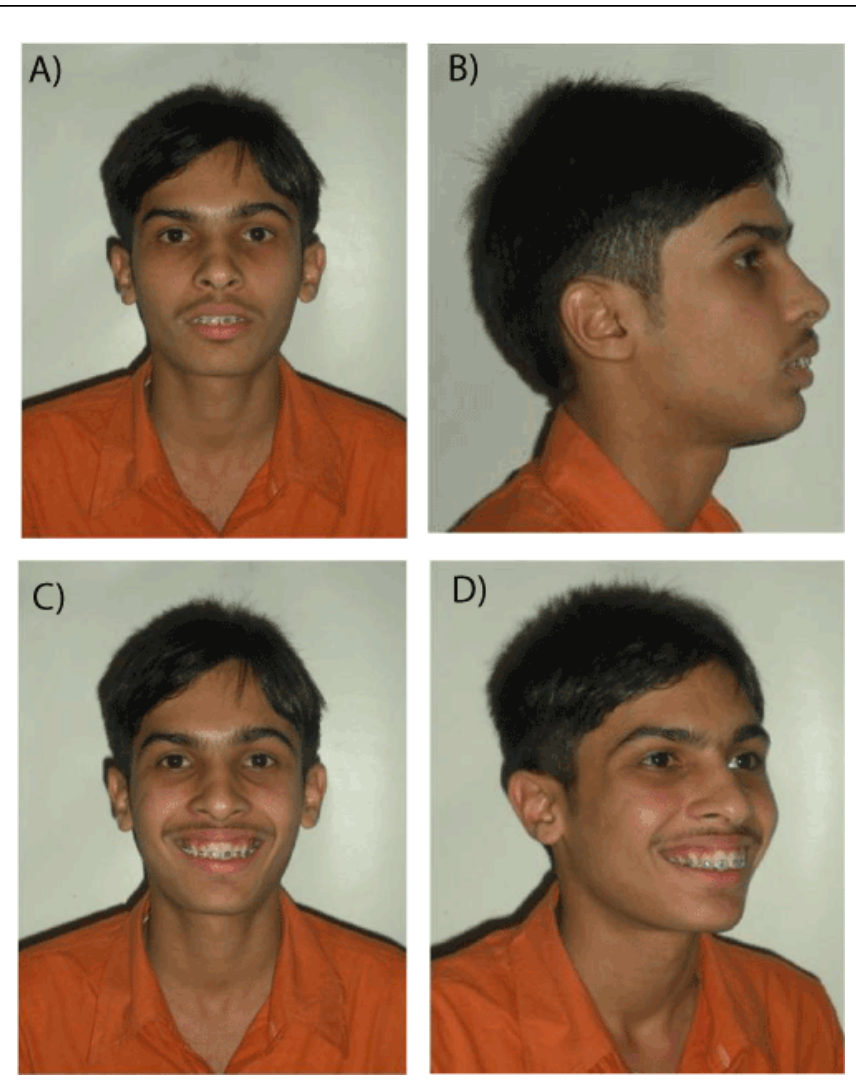

Figure 4: Pretreatment occlusal photographs

Surgery was too far elaborate and was instantly ruled out by the patient and the parents. The parents were further skeptical about tooth extraction and implant placement owing to the history of repeated apthous ulcer formation and finally opted for non extraction treatment with functional appliance.

Two appliances were thought over, being, twin block with combipull headgear or Forsus Fatigue Resistant Devive. The latter was finally zeroed owing to the relatively minimal amount of growth remaining (CVMI IV) and the ease and comfort it provided over any extraoaral-intraoral appliance combination, besides, minimizing the reliance on patient's compliance. Moreover, Forsus not only allowed the mandible to be positioned anteriorly, it also had a headgear like effect restricting the downward and forward growth of the maxilla which was a definite advantage in this case as it would limit, if not totally eliminate the gumminess seen in the smile due to excess maxilla.

The FRD is a three piece, semi-rigid telescoping system incorporating a superelastic nickel-titanium coil spring that can be assembled chair side in a relatively short amount of time. It is compatible with complete fixed orthodontic appliances and can be incorporated into pre-existing appliances. The FRD attaches at the maxillary first molar and onto the mandibular archwire, distal to either the canine or first premolar bracket. As the coil is compressed, opposing forces are transmitted to the sites of attachment.

Figure $5(\mathrm{a}-\mathrm{d})$ and $6(\mathrm{a}-\mathrm{c})$ shows his prefunctional extraoral and intraoral photographs respectively. 
Citation: Rai AK (2014) Determinant of a Successful Case: Clinical Changes or Cephalometric Readings? Class II Division 1 Correction Using Forsus Fatigue Resistant Device. Dentistry 4: 266. doi:10.4172/2161-1122.1000266

Page 4 of 7

At this stage, both the arches were consolidated using continuous ligation. The maxillary second molars were bonded and aligned. 16 degree lingual crown torque was added to minimize lower anterior flaring. Forsus FRD (35 mm) was placed for 11 months (Figure 7a-d).

Postfunctional settling phase lasted for about 5 months and involved the use of 0.016" stainless steel wires in both the arches and short class two elastics. The total treatment lasted for twenty nine months.

\section{Discussion}

This report presents the case of a patient with a class II division 1 malocclusion with both the mandible and the maxilla contributing to the problem. The compounding factor in this case was the limited cooperation from the patient's parents, missed appointments, repeatedly breakages and frequent bouts of apthous ulcers.

The treatment planning involved two steps, being, an initial step to align the arch (especially the maxillary) in anticipation of possible unlocking the mandible and allowing its potential growth to express by removing the interference from the retroclined upper lateral incisors. This was followed by a re evaluation phase to decide on functional, camouflage or surgical line of treatment in light of the growth remaining and in discussion with the patient and his parents.

Mulligan set up was used up in the alignment of incisors. A transpalatal arch was used to reinforce anchorage; however had to be removed due to the discomfort experienced by the patient. Further, no other anchorage reinforcement methods were accepted by the patient and the initial phase itself took around 13 months due to frequent breakages and missed appointments. This lack of cooperation was a major concern when revaluating the case. At the time of revaluation twin block with head gear was ruled out since the patient was already nearing his growth completion, besides distortion or breakage of extraoral appliance could have produced undesirable forces and had the potential for injuring the patient. Above all, it was highly unlikely that patient who had rejected a TPA would ever use it. This option was, as expected, rejected by the patient.

The second option of camouflage with upper premolars extraction and retraction using implants was rejected by the parents as they did not wanted to remove any tooth and were not ready to bear any additional cost of implant citing economic reasons.

The surgical option of premaxillary set back and impaction with rhinoplasty if needed was rejected by the patient for being aggressive and expensive. The final treatment plan to which the patient agreed involved fixed functional appliance on reassurance that the face would look better, however the correction of skeletal problem in toto may not be possible by this mean alone, was clearly explained to the patient and his parents. The patient was reluctant in the beginning but was willing to give it a try after being reassuring by foreshowing the improvement in his appearance by VTO. Forsus was a logical choice in this case as the appliance involved minimal patient's compliance, was relatively comfortable for the patient and easy to reassemble in case patient reports with breakage.
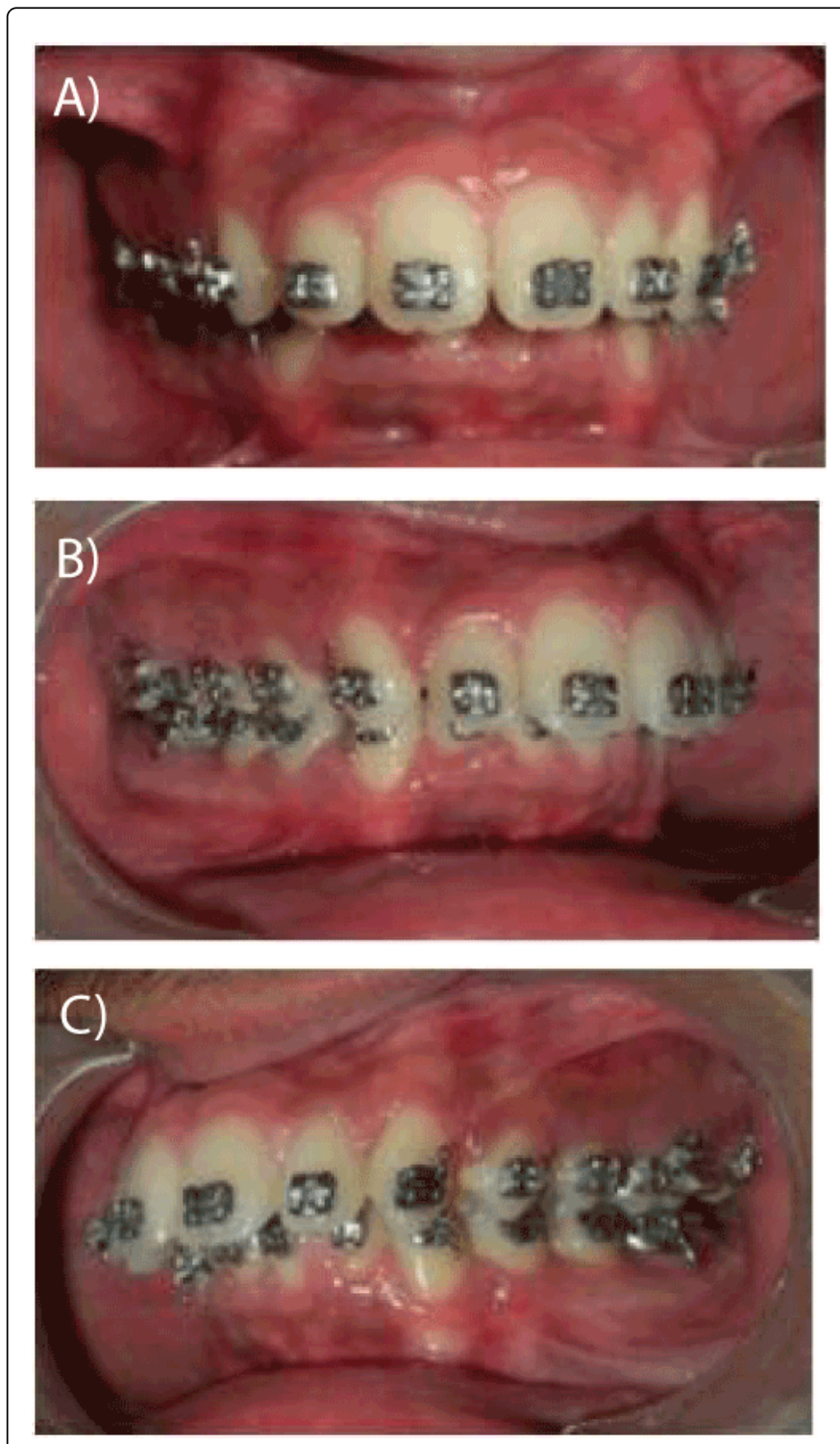

Figure 5: Prefunctional extraoral photographs 
Citation: Rai AK (2014) Determinant of a Successful Case: Clinical Changes or Cephalometric Readings? Class II Division 1 Correction Using Forsus Fatigue Resistant Device. Dentistry 4: 266. doi:10.4172/2161-1122.1000266

Page 5 of 7
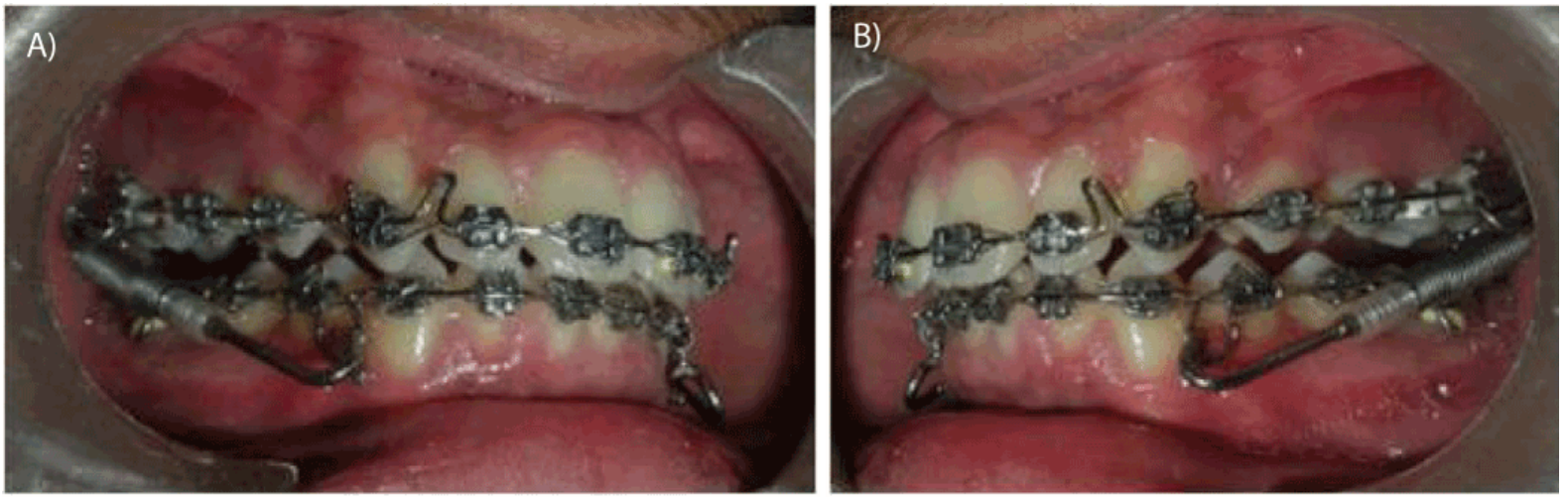

Figure 6: Prefunctional intraoral photographs

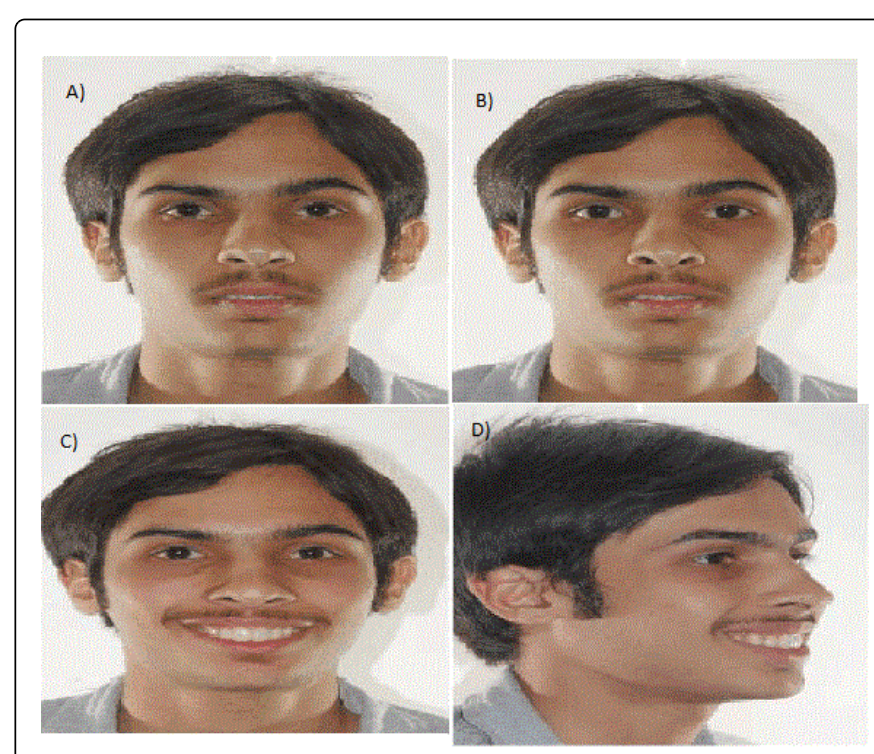

Figure 7: Extraoral photographs after placing Forsus FRD

Class II malocclusions treated using fixed dunctional appliance influence the jaws via the following mechanisms: remodeling of the mandibular condyle, remodeling of the glenoid fossa, repositioning the mandibular condyle in the glenoid fossa, and autorotation of the mandibular bone [1]. The effect on maxilla though not significant is like head gear, restricting the downward and forward growth of maxilla. Over the years, many fixed functional appliances have been used by orthodontists and only a few have shown well acceptance and favorable results on the patient [2,3]. Forsus FRD has long been proved to be one of the best treatment modality for class II malocclusion due to mandibular retrusion. It is capable of achieving class II correction in 3 to 6 months depending upon the baseline situation and the biological response [3].

The correction achieved is by a combination of skeletal and dental effects, $66 \%$ being dental and remaining $34 \%$ skeletal [4-7]. Significant improvement was noted in the soft tissue profile of the patient. Figures $8(a-d), 9(a-c)$ and $10(a, b)$ shows post treatment photographs of the patient. The clinical results achieved were highly satisfactory, both, to the patient and the parents. The patient's confidence was vividly seen in the broad end treatment photographs.

Figure 11a and $\mathrm{b}$ shows pretreatment, postfunctional and post treatment superimposition of patient.

\section{Treatment limitations}

The author would recognize the following limitations in the treatment of this patient, being, loss of anchorage seen in the upper arch leading to inability to retract the upper arch hence still leaving the patient with a gummy smile post treatment. However, the loss of anchorage was inevitable in this case as the anchorage reinforcement strategies were declined by the patient and repeated breakages and missed appointments protracted the treatment time.

The proclination of the lower incisors with the downward movement of the maxilla and the minimal change in mandibular growth. Most of the changes produced were dento alveolar than skeletal as much of valuable time when the patient still had considerable growth remaining was lost in the initial phase of treatment due to his non compliance.

However, this was an expected happening with the use of fixed functional appliance [4,5]. The proclination of lower incisors were seen as the adverse effect to the use of Forsus and occurred despite the addition of torque in the lower anterior segment of arch wire. However, could be considered acceptable considering the patient's growth pattern. 
Citation: Rai AK (2014) Determinant of a Successful Case: Clinical Changes or Cephalometric Readings? Class II Division 1 Correction Using Forsus Fatigue Resistant Device. Dentistry 4: 266. doi:10.4172/2161-1122.1000266
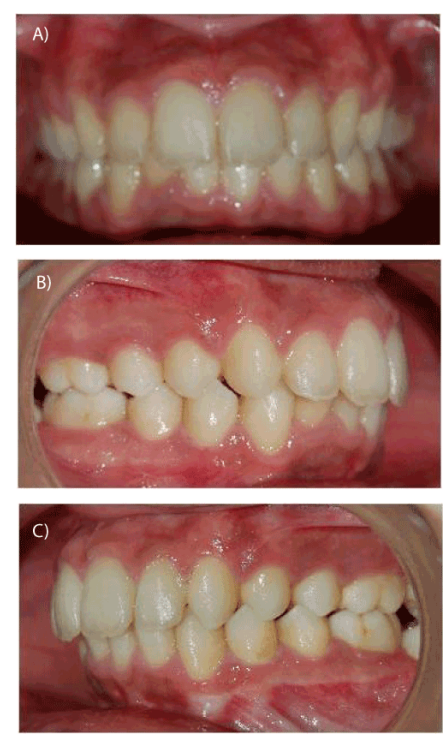

Figure 8: Post treatment photographs
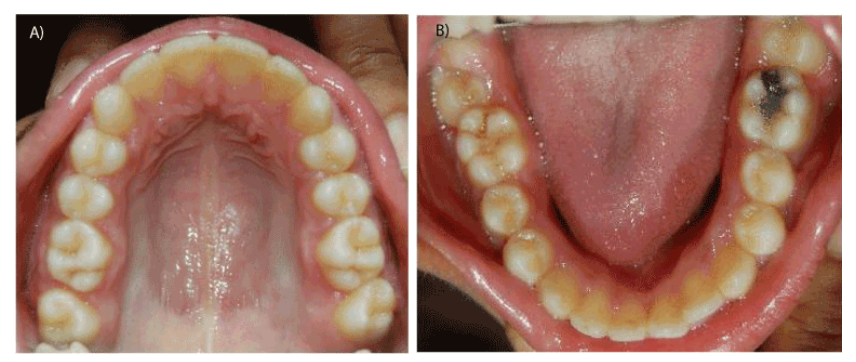

Figure 9: Post treatment photographs
PRE. POST EUNCTIONAL AND POST TREATMENT SUPERIMPOSITIONS Maxillary superimposition on ANS - PNS at ANS Mandibular superimposition on lower border at Symphysis

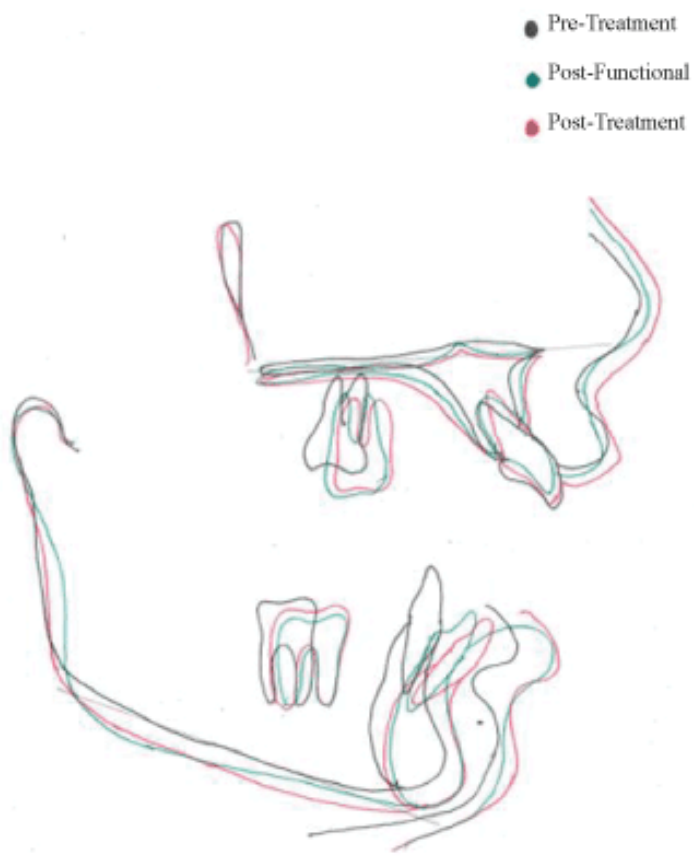

PRE. POST FUNCTIONAL AND POST TREATMENT SUPERIMPOSITIONS

Overall superimposition on Busion - Nasion at $\mathrm{CC}$ point

- Pre-Treatment

- Post-Functional

- Post-Treatment

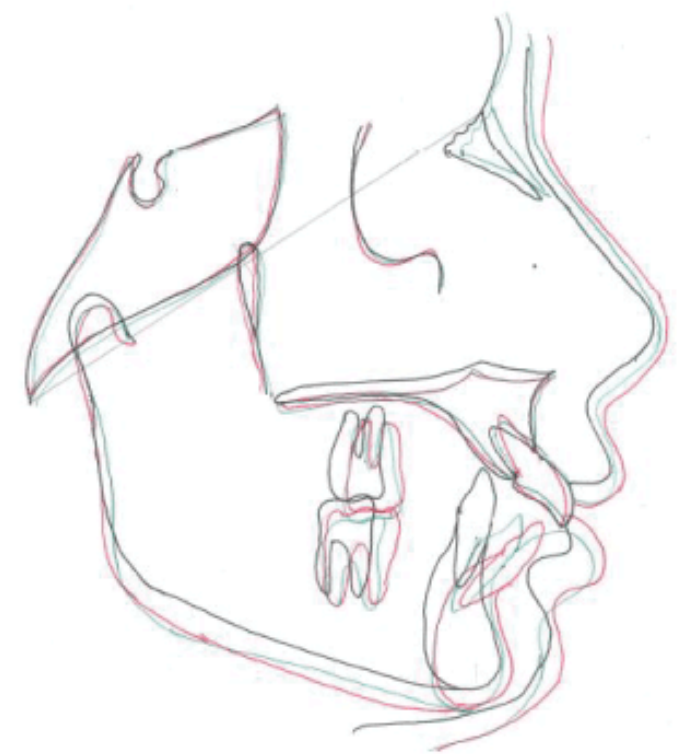

Figure 10: Post treatment photographs 
Citation: Rai AK (2014) Determinant of a Successful Case: Clinical Changes or Cephalometric Readings? Class II Division 1 Correction Using Forsus Fatigue Resistant Device. Dentistry 4: 266. doi:10.4172/2161-1122.1000266

Page 7 of 7

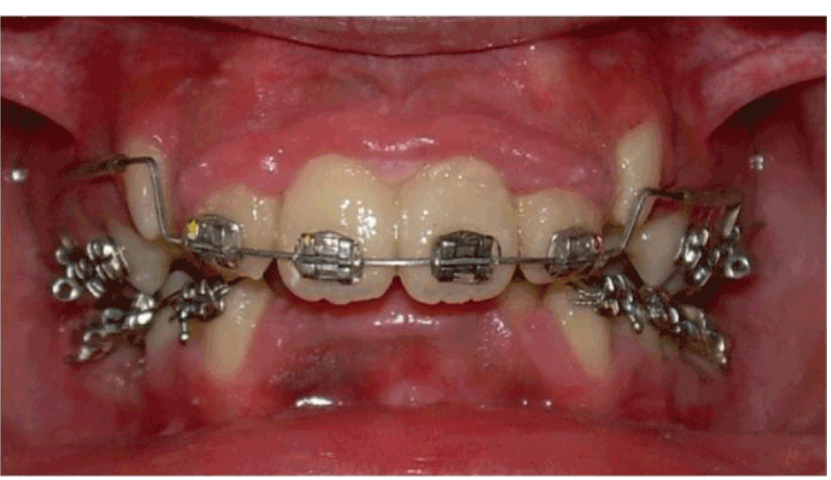

Figure 11: Pretreatment, postfunctional and post treatment superimposition

\section{Conclusion}

The result achieved in this case can be a bone of contention and can be called anyway between successful to acceptable to a failure. From a clinical prespective the result could be judged as a success. However, an in depth look at cephalometrics and numbers may even call it as a failure as the results were well short of cephalometric goals. The big question is: what does an orthodontist treats- faces or cephalograms? The evaluation of result in such cases, whether judged success or failure must be done cautiously. Due consideration must be given to a multitude of factors predominantly being the patient's expectations and their concerns. From a clinicians perspective, often, the cooperation from the patient, their accessibility to treatment and the financial constrains become a determining factor in deciding from available treatment options. The judgment of treatment results from being ideal to acceptable and yet, being judged successful should be based on what was planned and how much of it was achieved, corborating the dictum of 'begin with the end in mind'.

The patient in this case was satisfied with the result. Though the ideal cephalometric goals were not achieved and room of improvement remained the clinican also judged the case to be satisfactory since the facial changes achieved were marked and above all the cephalometric values, the patient walked out with a beeming smile.

\section{References}

1. Pancherz H, Ruf S, Kohlhas P (1998) "Effective condylar growth" and chin position changes in Herbst treatment: a cephalometric roentgenographic long-term study. Am J Orthod Dentofacial Orthop 114: 437-446.

2. Cope JB, Buschang PH, Cope DD, Parker J, Blackwood HO 3rd (1994) Quantitative evaluation of craniofacial changes with Jasper Jumper therapy. Angle Orthod 64: 113-122.

3. Karacay S, Akin E, Olmez H, Gurton AU, Sagdic D (2006) Forsus Nitinol Flat Spring and Jasper Jumper corrections of Class II division 1 malocclusions. Angle Orthod 76: 666-672.

4. Heinig N, Göz G (2001) Clinical application and effects of the Forsus spring. A study of a new Herbst hybrid. J Orofac Orthop 62: 436-450.

5. McNamara JA Jr, Bookstein FL, Shaughnessy TG (1985) Skeletal and dental changes following functional regulator therapy on class II patients. Am J Orthod 88: 91-110.

6. Jain AK, Patil AK, Ganeshkar SV, Sangamesh B, Chugh T (2012) Nonextraction treatment of skeletal class II malocclusion. Contemp Clin Dent 3: 334-337.

7. Hanoun A, Al-Jewair TS, Tabbaa S, Allaymouni MA, Preston CB (2014) A comparison of the treatment effects of the Forsus Fatigue Resistance Device and the Twin Block appliance in patients with class II malocclusions. Clin Cosmet Investig Dent 6: 57-63. 